\title{
SOCIAL ENGINEERING AS AN APPROACH FOR PROBING ORGANIZATIONS TO IMPROVE IT SECURITY: A CASE STUDY AT A LARGE INTERNATIONAL FIRM IN THE TRANSPORT INDUSTRY
}

\author{
Daniël van Liempd ${ }^{1}$, Arthur Sjouw ${ }^{1}$, Matthijs Smakman ${ }^{1,2}$ and Koen Smit ${ }^{1}$ \\ ${ }^{I}$ Institute for Information and Communication Technology, HU University of Applied Sciences Utrecht, 3584 CS Utrecht, \\ the Netherlands \\ ${ }^{2}$ Department of Communication Science, VU University Amsterdam, 1081 HV Amsterdam, the Netherlands
}

\begin{abstract}
This paper describes the external IT security analysis of an international corporate organization, containing a technical and a social perspective, resulting in a proposed repeatable approach and lessons learned for applying this approach. Part of the security analysis was the utilization of a social engineering experiment, as this could be used to discover employee related risks. This approach was based on multiple signals that indicated a low IT security awareness level among employees as well as the results of a preliminary technical analysis. To carry out the social engineering experiment, two techniques were used. The first technique was to send phishing emails to both the system administrators and other employees of the company. The second technique comprised the infiltration of the office itself to test the physical security, after which two probes were left behind. The social engineering experiment proved that general IT security awareness among employees was very low. The results allowed the research team to infiltrate the network and have the possibility to disable or hamper crucial processes. Social engineering experiments can play an important role in conducting security analyses, by showing security vulnerabilities and raising awareness within a company. Therefore, further research should focus on the standardization of social engineering experiments to be used in security analyses and further development of the approach itself. This paper provides a detailed description of the used methods and the reasoning behind them as a stepping stone for future research on this subject.
\end{abstract}

\section{KEYWORDS}

Social Engineering, Security awareness, Security analysis, Phishing

\section{INTRODUCTION}

In order for a business to determine their level of IT-security, a possible approach would be to carry out a security analysis. A security analysis can be seen as a measurement of the security posture of a system, employees or the organization (Abdel-Aziz, 2011). A method that can be used to analyse the human aspect of security, is to conduct a social engineering experiment. The experiment can be compared to penetration testing. Penetration testing is a process whereby someone imitates an adversary looking for security vulnerabilities, which allows the breach into a system or network (Abdel-Aziz, 2011). Rouse (2016) states that social engineering heavily relies on human interaction and often involves tricking people in breaking normal security procedures.

A social engineer performs what is also known as a "con game". Techniques such as appeal to vanity, appeal to authority and appeal to greed are often used in social engineering attacks. A lot of social engineering exploits simply rely on people's willingness to be helpful. For example, the attacker might pretend to be a co-worker who has some kind of urgent problem that requires access to additional network resources.

The benefits of targeting can be seen in the context of phishing. Since scammers have shifted their effort to increased targeting, they have also started to abandon the strategy of informing their victims since it has become less beneficial. Email scams have traditionally taken advantage of the negative emotions of people. Emotions such as greed, loneliness and fear (Jakobsson et al., 2016). 
Humans will always be the weakest link in the information security chain. The best security system in the world can be undermined by a single employee making a mistake. Phishing attacks resulted in nearly $\$ 6$ billion in losses in 2013 alone, spread out over some 450,000 separate cases. In 2015, Social engineering attacks were the most commonly used hacking technique and in 2016, sixty percent of enterprises were victims of some form of a social engineering attack (Warwick A, 2016). Therefore, social engineering can be a big threat to organisations.

This study was part of a broader security analysis of a large international organization in the transport sector. It was done in collaboration with the organization itself, as a select number of people was informed of the experiment. The security analysis was required because the organisation was unaware of their current state of security. The goal of the social engineering experiment was to determine the security vulnerabilities of the company and to raise awareness among the employees. Coming from these challenges in practice, the following research question has been formulated for this study:

In what way can social engineering contribute to improve the IT security of a company?

The remainder of this paper is structured as follows. First, the background and related work will be described. After that, the research method will be explained, followed by the results and conclusions. The document ends with the discussion and future research.

\section{BACKGROUND AND RELATED WORK}

Several parts of social engineering have been studied by researchers before (e.g. Greavu-Şerban \& Şerban, 2014 and Rao \& Nayak, 2014). Social engineering can be divided into several elements, such as: phishing, vishing, baiting, and tailgating (Rao \& Nayak, 2014), making social engineering a complex, interdisciplinary phenomenon. However, the elements of social engineering have a common goal, being: to trick the target into doing something (Greavu-Şerban \& Şerban, 2014). In the field of information technology this usually means acquiring privacy sensitive information such as login credentials. One of the most common forms of social engineering is Phishing (Rao \& Nayak, 2014).

Phishing involves sending an email to someone, while pretending to be someone else. The email usually contains a link to a website that the engineer recreated. The recipients are then asked to click on the link and perform certain actions that might result in the victim compromising their personal information like their login credentials (Tiwari, 2017). In order to increase the rate of success, the social engineer often has an address that closely resembles a legitimate address, either close to one of the organisation or an external one. If the organization's domain is called theorganization.com, it's a completely valid option for the attacker to buy the domain theorganization.org as these domain names can usually be bought for a low price, provided that it's available. Even if the recipient notices that the extension is different than usual, there's still a good chance they'll fall for it. Because it's easy to send these emails to hundreds of people, the attacker doesn't need a hit on every single one. For many use cases just a couple of hits are sufficient (Rao \& Nayak, 2014).

Vishing, short for "voice phishing", is similar to phishing. Instead of using an email to contact the target, Vishing involves calling the target. Attackers often assume the role of an employee of a legitimate organization such as a bank, claiming that they encountered an issue with the targets account. These calls are also often automated, guiding the target to provide privacy sensitive information through automated questions. Data acquired this way is then used for either identity theft or used for follow up attacks (Rao \& Nayak, 2014).

Tailgating is when the social engineer is physically at a location and tries to gain access to a restricted building where RFID authentication or another form of electronic barrier is present by using the help of an authorized person (Tiwari, 2017). A possible follow up after tailgating could be baiting.

Baiting can be compared to an approach that is known as the trojan horse. In the case of baiting, a social engineer leaves infected USB drives or optical disks at certain places with the hope of someone using it on their device out of curiosity (Tiwari, 2017). The USB drives that are used in baiting could be working similarly to a USB Rubber Ducky. Brody (2017) describes the USB Rubber Ducky as an innocent USB drive that can disguise itself as a keyboard when plugged into a computer. As it is registered as a keyboard it can execute pre-programmed commands. Brody (2017) also states that it is not difficult to create the commands. This is because the manufacturer of the USB Rubber Ducky created their own syntax and has made up a full list of commands and what they do. 
There are also other types of social engineering aside from phishing, vishing, tailgating and baiting. Those types are Quid Pro Quo and Pretexting resulting in a total of 6 types (Bisson, 2015).

Nadeem (2015) states that Quid Pro Quo are attacks that make the promise of giving a benefit in exchange for information. An example of this could be free clothing or access to an online game in exchange for your login credentials.

For the definition of pretexting Nadeem (2015) states that it is another form of social engineering, where attackers focus on creating a fabricated scenario that can be used to steal their victim's personal information. It commonly takes the form of a scammer that pretends certain bits of information is needed in order to confirm the identity of the victim.

How these methods were used during the research, will be explained in the next chapter.

\section{RESEARCH METHOD}

The goal of the social engineering experiment was to determine the security vulnerabilities of the company and to raise awareness among the employees. The research method is considered to be quantitative, because the project team gathered the data by pinpointing the amount of employees that fell for the social engineering attack and analyse the data to determine the level of awareness based on the amount of victims. A literature study is conducted beforehand to gain knowledge about the different types of social engineering attacks in order to determine which type(s) would be the most effective to gain results.

To carry out the social engineering experiment, three techniques were used. The first technique was to send phishing emails to the employees as well as the system administrators. Two emails were written, one for each target group as the intended goal was different between the two. The purpose of sending the mail to the employees was to prove that they can be tricked by an attacker to raise security awareness. The purpose of sending an email to the system administrators was to trick them into sharing their password so that the firewall could be by bypassed and the network breached. The second technique was to leave a rubber ducky on an employee's desk. The goal of that technique was the same as sending phishing emails to the employees: to raise awareness. The third technique, tailgating, is directly related to the rubber duckies in this case as tailgating was used to reach the target area.

A social engineering attack can only be successful if the employee thinks you are trustworthy (Rao \& Nayak, 2014). The more an attacker knows about the company, the more methods the attacker can use in order to gain trust when executing the experiment. For example, before sending out the emails, the researchers bought a domain with a name that looks a lot like the company's name. Therefore, the phishing emails could be sent from the address: management@example-company.com, which looks a lot more trustworthy than hacker@ gmail.com. Normally an attacker would also have to find the email addresses of their targets, but due to the limited time available, the project team's contact within the company provided the addresses.

For the experiment of sending phishing emails to the employees with low IT-knowledge, a couple of methods were used in order to make the email trustworthy. As an excuse, the employees were asked to fill in a survey by clicking on a hyperlink. However, when they clicked the link they were instantly redirected to a website set up for the experiment. On that website, the employees received a pop-up that said they had been fooled and that they should be aware of these kinds of emails. The website also showed the employees what kind of information an attacker can retrieve when a site is visited, for instance, the web browser and IP address. This information was also stored in a database in order to keep track of how many people had truly clicked the link, just in case one person had clicked the link multiple times. In total, 26 employees received this email.

The experiment of sending emails to the system administrators was partly similar. Once again some of the same procedures were used in order to make the email appear trustworthy. The email contained an explanation of how the login page for the firewall had been renewed and they had to follow the link to the new page. However, when they clicked the hyperlink, they were not redirected to the webpage set up for the employees. Instead, they were redirected to a webpage that was an exact copy of the normal firewall login page they used. When they entered their username and password, it was once again saved in the database. Logically, when they entered their correct user data, they would just receive an error that it was incorrect. They might get suspicious when they enter their password correctly multiple times, therefore the webpage was configured to present a time-out after three failed attempts. In total, 5 employees received this email. 
Executing the rubber ducky experiment was different from sending out the phishing emails. The rubber ducky looked like a regular USB stick. However, when plugged into a computer, it automatically runs the software that it contains. In case of an actual attacker, it might contain a virus that infects all devices on the computer's network. The rubber duckies used in this experiment were fairly harmless, as they only redirected the user to the aforementioned webpage. That web page displayed a pop-up similar to the one used for the phishing emails. Once again, the information of the computer opening our website was stored in our database. Only two rubber duckies were used for this experiment, due to limited resources. One of the methods we came up with, was putting the rubber ducky on top of a pile of paper that employee A gave to employee B. Therefore, it looked like the rubber ducky belonged to employee A.

Part of executing this experiment was getting onto the work floor of the company in order to circulate the rubber ducky's. The difficulty of this activity can vary depending on the physical security of the target organization. In order to measure the level of security a company has, it has to be tested if an attacker can physically enter the work floor. If that is possible, there are more risks than only placing rubber duckies, such as theft of documents or devices which can also contribute to breaching the IT security.

The more information a hacker has, the more trustworthy the attack can look. Therefore, employees should not only be aware of giving their password or installing a virus, but they should also be careful when sharing personal or company information to strangers.

\section{RESULTS}

The infiltration of the building was successful, but it was performed later in the day. The building was quite empty. Once on the right floor, it was a matter of tactically placing the USB drives. This was done by putting the USB stick on a document, which came from another employee, thus making it look like it was part of the documents.

Our experiment emails might have been intercepted by Microsoft's spam filter. Therefore, the researchers first checked, by sending an email to themselves, if these emails would end up in the spam folder. This has been tested on both Outlook and Gmail. Both services forwarded the emails and did not mark them as spam.

In Table 1 the results of the experiment are shown. The results are divided per target group. They are further distinguished by the number of visits (how many times someone became victim), unique visits and whether the victim was using the company network or not.

Table 1. Results social engineering experiment

\begin{tabular}{|c|c|c|c|}
\hline Origin & Visits & Unique visits & From company network \\
\hline USB drives & 3 & 3 & 1 \\
\hline Administrator mail & 7 & 6 & 0 \\
\hline Employee mail & 5 & 4 & 1 \\
\hline
\end{tabular}

The USB drives had fewer visits than expected, most likely due to the number of USB drives there were circulated. After asking what has happened to the USB's we learned that one of them was taken home with an employee, however, this employee put it away and forgot about it. The other one was passed around the office after the first employee became a "victim", but was lost after the second pass. However, according to the logs, the USB drive seems to have been inserted at someone's home computer.

Sending the emails led to suspicion among some of the employees. One of them sent out an email to all other employees on the floor, telling them not to click the link. That employee also notified the IT department and asked for them to inspect the email. The IT department answered within an hour and informed the employees that the link was malicious. However, in that same amount of time, some employees had already clicked on the link. 
Table 2. Event overview

\begin{tabular}{|c|c|}
\hline Event & Date \\
\hline USB drives were planted & $20 / 12 / 2017$ at $3: 15 \mathrm{pm}$ \\
\hline USB drive was inserted from within the company network & $21 / 12 / 2017$ at $8: 05$ am \\
\hline Mails were sent to administrators and employees & $21 / 12 / 2017$ at $12: 52 \mathrm{pm}$ \\
\hline Mail link was clicked from within the company network & $21 / 12 / 2017$ at $12: 55 \mathrm{pm}$ \\
\hline First warning mail telling all employees to be careful as it might be spam & $21 / 12 / 2017$ at $12: 57 \mathrm{pm}$ \\
\hline Second warning mail telling all employees that it is spam and not to click it & $21 / 12 / 2017$ at $1: 04 \mathrm{pm}$ \\
\hline Mail link was clicked from outside the company network & $21 / 12 / 2017$ at $2: 46 \mathrm{pm}$ \\
\hline Mail link was clicked from outside the company network & $21 / 12 / 2017$ at $2: 47 \mathrm{pm}$ \\
\hline Mail link was clicked from outside the company network & $21 / 12 / 2017$ at $6: 53 \mathrm{pm}$ \\
\hline USB drive was inserted from outside the company network & $23 / 12 / 2017$ at $9: 28 \mathrm{pm}$ \\
\hline Mail link was clicked from outside the company network & $25 / 12 / 2017$ at $9: 26$ am \\
\hline Firewall link was clicked from outside the company network & $06 / 01 / 2018$ at $2: 18 \mathrm{pm}$ \\
\hline Firewall link was clicked from outside the company network & $16 / 01 / 2018$ at $1: 38 \mathrm{pm}$ \\
\hline Firewall link was clicked from outside the company network & $16 / 01 / 2018$ at $9: 16 \mathrm{pm}$ \\
\hline Firewall link was clicked from outside the company network & $26 / 01 / 2018$ at $10: 34 \mathrm{pm}$ \\
\hline Firewall link was clicked from outside the company network & $29 / 01 / 2018$ at $10: 59 \mathrm{am}$ \\
\hline Firewall link was clicked from outside the company network & $29 / 01 / 2018$ at $1: 19 \mathrm{pm}$ \\
\hline Firewall link was clicked from outside the company network & $30 / 01 / 2018$ at $1: 49 \mathrm{pm}$ \\
\hline USB drive was inserted from outside the company network & $02 / 02 / 2018$ at $2: 42 \mathrm{pm}$ \\
\hline
\end{tabular}

As described earlier, the employee email and administrator email were different and they didn't get both emails. Once the employees got a warning, the administrators didn't. The administrators had no clue on what was going on with the other employees and so the email was not flagged as suspicious for them. Thus, resulting in 6 unique visits. Of those 6 visitors, 2 users entered their login credentials trying to log in. They both entered them twice in succession, falling for the attack twice. That information, allowed the research team to infiltrate the network and have the possibility to disable or hamper crucial processes. Figure 1 gives an overview of the total process of the social engineering experiment. 


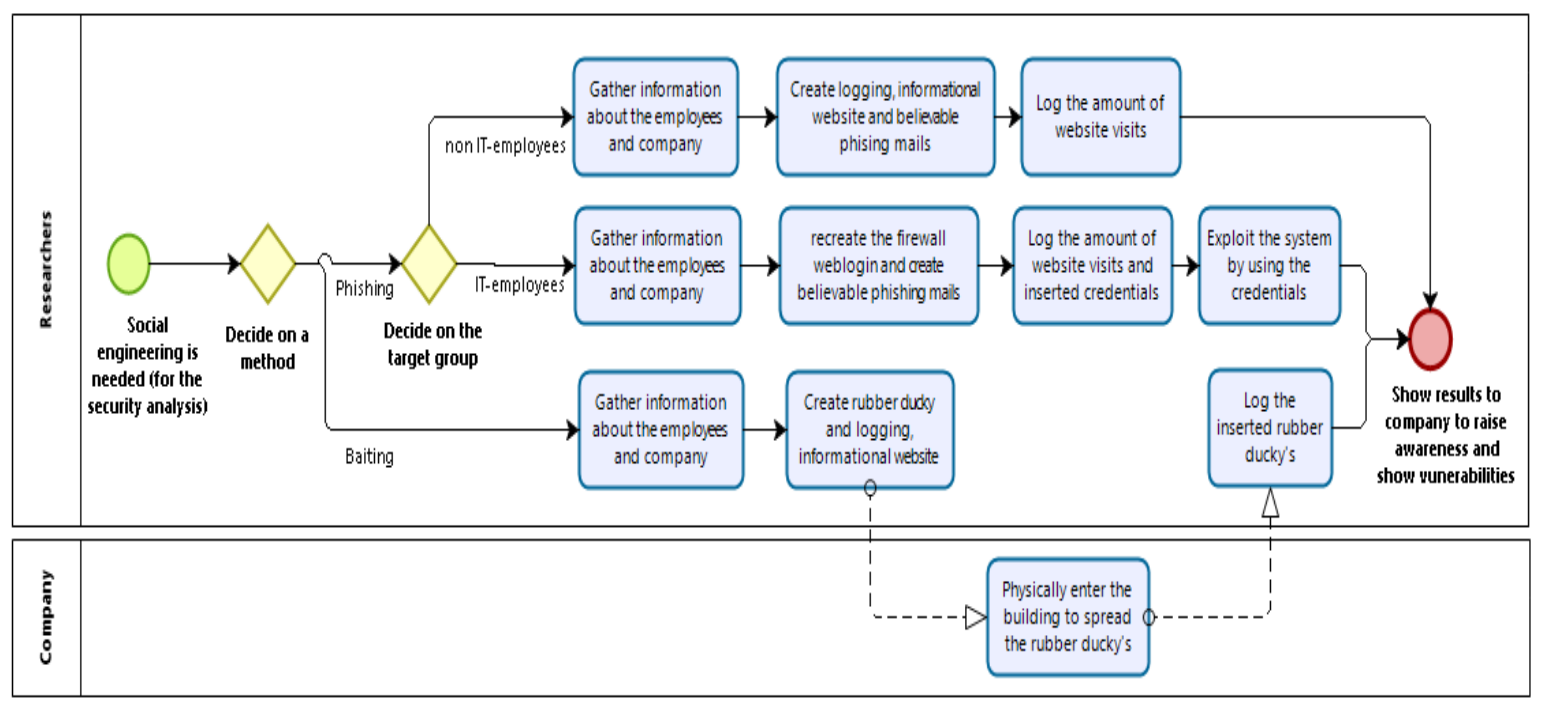

Figure 1. The Process overview

The managers, belonging to the Dutch branch of the company, had not been in the loop and were notified once the research was completed. As they didn't have any information until the research was completed, they became quite shocked. It was unknown to them that the company was having such risks.

\section{CONCLUSION}

The goal of the research was to determine the security vulnerabilities of the company and to raise awareness among the employees. The research question that has to be answered is as follows:

In what way can social engineering contribute to improve the IT security of a company?

The expectations regarding the results of the conducted experiment were that employees responsible for the network would not easily fall for these attacks. It was assumed that they would be aware of these types of attacks. The research team did expect some of the employees to be tricked by the phishing emails and the rubber ducky's, because of the unawareness the employees had showed regarding IT security.

However, the researched organization became a victim of the social engineering attack. The two Rubber Ducky's were inserted three different times and four out of 26 employees had visited the website by clicking the link in the phishing mail. Thereby proving that the employees were vulnerable for phishing and baiting. The most valuable group, the administrators, were tricked and filled in their credentials. With that information the research group was able to infiltrate the company. Therefore, the attack was successful and the organization was vulnerable.

Based on the results, we can conclude that social engineering proved to be an effective method to reveal the vulnerabilities of the company. The biggest reason the social engineering attacks was effective, is because the employees think that they are safe. They believe their company is not a good target for attackers. When employees are not aware that they might be are target, they are more likely to make mistakes. Attackers can use those mistakes to infiltrate a company, as demonstrated during our research.

Revealing the organization's vulnerabilities had an interesting effect. It resulted in a call to action for the organization and its employees to fix the security issues that were discovered. Part of fixing the security issues, was giving the employees an awareness training and improving components of the infrastructure that were exposed due to the attack. However, this method is not exclusive to this particular case and can be used for any organization that shows weakness in terms of security awareness. Besides, social engineering and possible countermeasures are easy to understand, even for employees with low IT knowledge which makes it a good option for raising awareness (Alexander, 2016). Therefore, social engineering can contribute to improve the IT security of a company by raising awareness and showing the vulnerabilities of a company. 


\section{DISCUSSION \& FUTURE RESEARCH}

Each research study has its limitations. First of all, more research could have been conducted on different aspects of social engineering. There are more methods we could have included to diversify our results. This could also give us an opportunity to compare the success rate of the used methods. One of these methods is putting rubber ducky's directly in the computers, instead of just leaving them on employee's desk. This method was not chosen, because it did not contribute to the goal of proving that the employees were not aware of security threats. Another unused method Vishing, because it is an aggressive method and carried a risk of exposure.

The methods that were used also had some flaws. Only two rubber duckies were used, so the sample size is small. Because of time limitations, the email addresses were provided by the company so the step of obtaining them was significantly easier compared to a situation in which an attacker needs to obtain these addresses themselves. The login credentials obtained through the phishing emails also wouldn't be of any use without access to the firewall itself. Its IP address was provided by the company as well and therefore the address didn't have to be acquired through other methods. Since the firewall was accessible from outside the organization's network, it would only be a matter of time for an attacker to discover its IP address.

Future research could include exploring the different social engineering methods. This would provide insight in which methods are more effective. This could eventually lead to creating a standard approach to social engineering. That standard could be integrated with future security analyses.

The results of those experiments must be compared in future research so that the effectiveness of different type(s) of social engineering attacks can be determined per specific context. This will help organizations that want to evaluate their IT-security (awareness) in that they will know what kind of social engineering experiments they will need to conduct in their context.

\section{REFERENCES}

Abdel-Aziz, A. (2011). Scoping Security Assessments - A Project Management Approach. In 2011 SANS Institute (pp. 2-4)

Alexander, M. (2016, April 30). Methods for Understanding and Reducing Social Engineering Attacks. In 2015 SANS Institute (pp. 23-25)

Bisson, D. (2015, March 23). 5 Social Engineering Attacks to Watch Out for. Retrieved June 4, 2018, from https://www.tripwire.com/state-of-security/security-awareness/5-social-engineering-attacks-to-watch-out-for/

Brody, H. (2017, August 29). USB Rubber Ducky Tutorial: The Missing Quickstart Guide to Running Your First Keystroke Payload Hack [Blog post]. Retrieved June 4, 2018, from https://blog.hartleybrody.com/rubber-ducky-guide/

Bowers, T. (2017, August 17). How hackers find their targets - Data Breach Resolution. Retrieved June 11, 2018, from http://www.experian.com/blogs/data-breach/2011/09/06/how-hackers-find-their-targets/

Defranzo, S. E. (2011, September 16). What's the difference between qualitative and quantitative research? Retrieved May 12, 2018, from https://www.snapsurveys.com/blog/qualitative-vs-quantitative-research/

Greavu-Şerban, V., \& Şerban, O. (2014). Social Engineering a General Approach. In 2014 Informatica Economică vol. 18 , no. 2 (pp. 6-8)

Jakobsson, M., Dhiman, M., Ferreira, A., Jafarikhah, S. T., McCoy, D., Park, Y., Yen, T. F. (2016). Understanding Social Engineering Based Scams. Cham, Switzerland: Springer Nature. In 2016 Springer-verlag New York (pp. 29-30)

McLeod, S. (2017). Qualitative vs. Quantitative. Retrieved June 9, 2018, from https://www.simplypsychology.org/qualitative-quantitative.html

Nadeem, M. S. (2015, November 25). Social engineering: Quid Pro Quo attacks [Blog post]. Retrieved June 4, 2018, from https://blog.mailfence.com/quid-pro-quo-attacks/

Nadeem, M. S. (2018, November 13). Social Engineering: What is pretexting? [Blog post]. Retrieved June 2, 2018, from https://blog.mailfence.com/pretexting/

Rao, U. H., \& Nayak, U. (2014). Social Engineering. In U. H. Rao, \& U. Nayak (Eds.), An Introduction to Information Security. In 2014 Apress Open (pp. 316-319). 
Rao, U. H., \& Nayak, U. (2014). Social Engineering. In U. H. Rao, \& U. Nayak (Eds.), An Introduction to Information Security. In 2014 Apress Open (pp. 318-318).

Rao, U. H., \& Nayak, U. (2014). Social Engineering. In U. H. Rao, \& U. Nayak (Eds.), An Introduction to Information Security. In 2014 Apress Open (pp. 290-290).

Rouse, M. (2016, February). social engineering. Retrieved May 12, 2018, from https://searchsecurity.techtarget.com/definition/social-engineering

Warwick, A. (2016) Social engineering confirmed as top information security threat. Retrieved February 5, 2019, from https://www.computerweekly.com/news/4500273577/Social-engineering-confirmed-as-top-information-securitythreat

Tiwari, A. (2017, February 28). What Is Social Engineering? What Are Different Types of Social Engineering Attacks? Retrieved May 12, 2018, from https://fossbytes.com/what-is-social-engineering-types-techniques/ 